\title{
SPIKE AND WAVE EEG ABNORMALITIES IN ADHD
}

Investigators at Istanbul University, Turkey, studied sleep EEGs in 134 patients with ADHD. All patients had spike and wave paroxysms that changed with age. Half of the patients had seizures, and 38 had the EEG findings of benign focal epilepsy of childhood. Spike and wave activity evolves in time, and EEG discharges are related to neuropsychiatric symptoms even in the absence of epilepsy. It is recommended that sleep EEG be considered in all cases of ADHD (Altunel A, Altunel EO, Sever A. Electroencephalogram in attention deficit hyperactivity disorder: spike and wave paroxysms, foci, and seizures. J Clin Neurophysiol 2013 Aug;30(4):357-61).

COMMENT. This study confirms previous reports of a high incidence of epileptiform abnormalities in the EEG of children with ADHD.

Valproate, EEG paroxysmal abnormality, and ADHD behavioral improvement. Of $46 \mathrm{ADHD}$ children treated with valproate sodium, 16 showed EEG paroxysmal abnormality (PA). The EEG improved in 5 of 8 with frontal PA and 3 of 5 with rolandic PA. EEG improvement with AED treatment showed a high correlation with behavioral improvements. (Kanemura $\mathrm{H}$, et al. EEG improvements with antiepileptic drug treatment can show a high correlation with behavioral recovery in children with ADHD. Epilepsy Behav 2013 Jun;27(3):443-8).

Sleep deprived EEG should be considered in children with ADHD, especially those with altered awareness or a family history of epilepsy. The AAP guideline that excludes the EEG in the workup of children with ADHD may need modification.

\section{MUSCLE DISORDERS}

\section{PROGRESSIVE SCOLIOSIS IN ULLRICH CONGENITAL MUSCULAR DYSTROPHY (UCMD)}

Investigators at National Center of Neur \& Psych, Kodaira, Tokyo, conducted a nationwide survey of 5442 neurologists regarding the natural history of UCMD. Among 40 patients with UCMD, 33 had collagen VI deficiency. Cobb angle was $>30^{\circ}$ at age 9.9 yrs, and \% vital capacity (VC) was decreased with age, with severe respiratory dysfunction before puberty. Scoliosis surgery in 3 patients at ages 5, 9 and 10 years resulted in well-maintained \% $\%$ in the youngest patient. Twenty-five (81\%) of 31 patients walked independently by age 1.7 years but lost this ability by age 8.8 yrs. Six patients never walked. Age of onset varied, but scoliosis and respiratory dysfunction progressed rapidly once started. (Yonekawa T, Komaki H, Okada M, et al. Rapidly progressive scoliosis and respiratory deterioration in Ullrich congenital muscular dystrophy. J Neurol Neurosurg Psychiatry 2013 Sep;84(9):982-8). (Response: Dr I Nishina. E-mail:nishino@ncnp.go.jp).

COMMENT. UCMD is manifested by proximal joint contractures and muscle weakness, distal joint hyperlaxity, scoliosis, and respiratory failure. Mutations in a COL6A gene are the cause. 